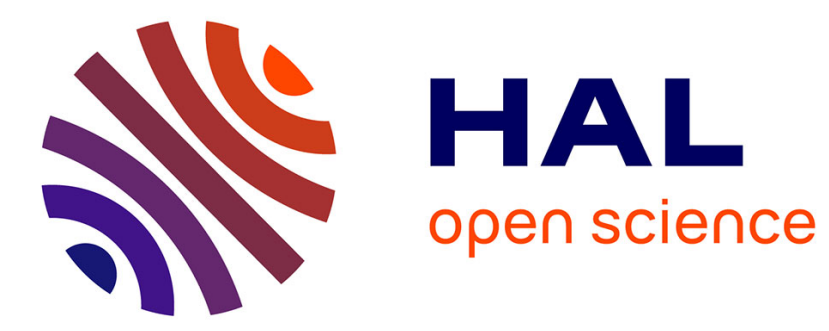

\title{
Occlusion-free Blood Flow Animation with Wall Thickness Visualization
}

Kai Lawonn, Sylvia Glasser, Anna Vilanova, Bernhard Preim, Tobias Isenberg

\section{To cite this version:}

Kai Lawonn, Sylvia Glasser, Anna Vilanova, Bernhard Preim, Tobias Isenberg. Occlusion-free Blood Flow Animation with Wall Thickness Visualization. IEEE Transactions on Visualization and Computer Graphics, 2016, 22 (1), pp.728-737. 10.1109/TVCG.2015.2467961 . hal-01178054

\section{HAL Id: hal-01178054 \\ https://inria.hal.science/hal-01178054}

Submitted on 17 Jul 2015

HAL is a multi-disciplinary open access archive for the deposit and dissemination of scientific research documents, whether they are published or not. The documents may come from teaching and research institutions in France or abroad, or from public or private research centers.
L'archive ouverte pluridisciplinaire HAL, est destinée au dépôt et à la diffusion de documents scientifiques de niveau recherche, publiés ou non, émanant des établissements d'enseignement et de recherche français ou étrangers, des laboratoires publics ou privés.

$$
\text { Copyright }
$$




\title{
Occlusion-free Blood Flow Animation with Wall Thickness Visualization
}

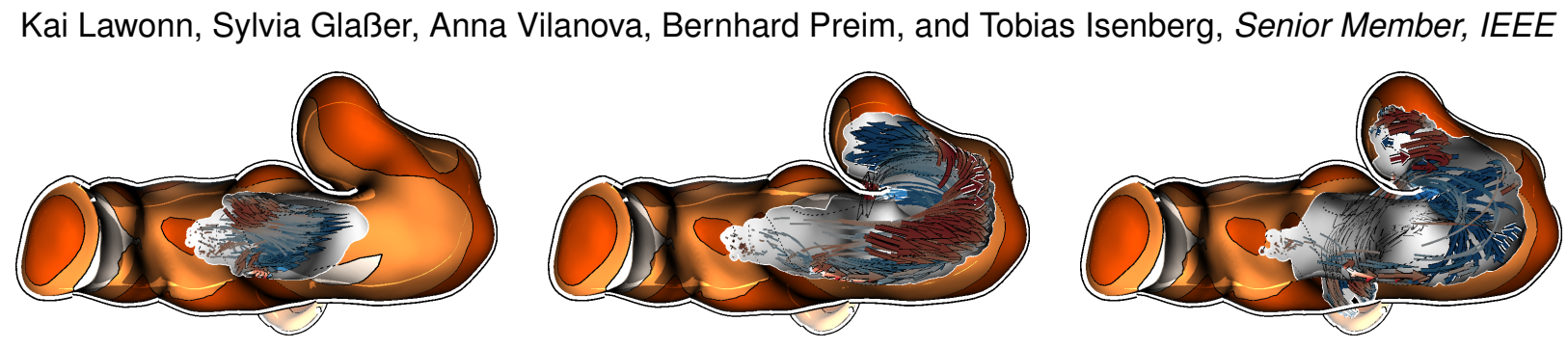

Fig. 1. Three subsequent time steps of the animated pathlines in a vessel. The cutaway technique facilitates insight into the vessel during the animation of the blood flow. Furthermore, arrow glyphs represent the flow's pathlines.

\begin{abstract}
We present the first visualization tool that combines pathlines from blood flow and wall thickness information. Our method uses illustrative techniques to provide occlusion-free visualization of the flow. We thus offer medical researchers an effective visual analysis tool for aneurysm treatment risk assessment. Such aneurysms bear a high risk of rupture and significant treatment-related risks. Therefore, to get a fully informed decision it is essential to both investigate the vessel morphology and the hemodynamic data. Ongoing research emphasizes the importance of analyzing the wall thickness in risk assessment. Our combination of blood flow visualization and wall thickness representation is a significant improvement for the exploration and analysis of aneurysms. As all presented information is spatially intertwined, occlusion problems occur. We solve these occlusion problems by dynamic cutaway surfaces. We combine this approach with a glyph-based blood flow representation and a visual mapping of wall thickness onto the vessel surface. We developed a GPU-based implementation of our visualizations which facilitates wall thickness analysis through real-time rendering and flexible interactive data exploration mechanisms. We designed our techniques in collaboration with domain experts, and we provide details about the evaluation of the technique and tool.
\end{abstract}

Index Terms-Medical visualization, aneurysms, blood flow, wall thickness, illustrative visualization.

\section{INTRODUCTION}

An aneurysm is a blood-filled expanded bulge in the wall of a blood vessel that may occur everywhere in the arterial system, predominantly at the abdominal aorta and at the cerebral arteries. Inherent to aneurysms is the risk of rupture which may result in fatal consequences for the patient. In this paper we focus on cerebral aneurysms, i.e., those that occur in the brain. Cerebral aneurysms result from a pathological weakness in the vessel wall, causing a dilation of the vessel structure. The initiation and outcome of such a cerebrovascular disease comprises different factors such as hemodynamic information $[6,27]$ and the wall thickness of the vessel wall [19]. Treatment options have to be evaluated compared to the aneurysm's rupture risk because treatment may cause a rupture as well. Current research in medical imaging has made great advances, in particular for intravascular data for (the larger) cardiac vessels. Moreover, wall thickness assessment will likely be possible for human cerebral arteries in the near future. Visualization techniques and tools are thus essential that are capable of presenting all this information in an integrated view. Furthermore, it is vital to incorporate the behavior of the time-dependent flow as well. The relation between

- Kai Lawonn is with the University of Magdeburg, Germany; TU Delft, Netherlands; Inria, France; and Research Campus STIMULATE. E-mail: lawonn@isg.cs.uni-magdeburg.de.

- Sylvia Glaßer is with the University of Magdeburg, Germany, and Research Campus STIMULATE.E-mail: glasser@isg.cs.uni-magdeburg.de.

- Anna Vilanova is with TU Delft, Netherlands. E-mail: A.Vilanova@tudelft.nl.

- Bernhard Preim is with the University of Magdeburg, Germany, and Research Campus STIMULATE. E-mail: bernhard@isg.cs.uni-magdeburg.de.

- Tobias Isenberg is with Inria, France. E-mail: tobias.isenberg@inria.fr.

Manuscript received 31 Mar. 2015; accepted 1 Aug. 2015; date of publication xx xxx 2015; date of current version xx xxx 2015.

For information on obtaining reprints of this article, please send

e-mail to:tvcg@computer.org. the vessel morphology and the near-wall flow is particularly important. Near-wall flow may lead to a pathological vessel dilation, which may increase the risk of a new rupture at locations where a former rupture occurred. The main challenge is, therefore, to combine visualization techniques that simultaneously illustrate both the outer wall (i.e., the border between vessel wall and surrounding tissue) and the inner wall (i. e., the lumen and vessel wall border) to allow physicians to get an impression of the wall thickness.

Current visualization solutions can either illustrate the blood flow with the surrounding vessel or depict the wall thickness without integrated blood flow, but a combined visualization was not possible before. Therefore, we are the first who present a visualization approach tailored to the occlusion challenges by presenting wall thickness with blood flow. We introduce a visualization technique based on cutaway surfaces such that occlusion problems with respect to the internal blood flow are resolved. We integrate this technique into a framework that we developed in collaboration with experts from neuroradiology, computational fluid dynamics, and medical illustration. Furthermore, we focus on illustrating the blood flow by simultaneously depicting it with the vessel wall as shown in Fig. 1. We developed the visualization technique and the framework based on hand-drawn images provided by medical researchers. These images indicate how the experts would envision the tool, and how they would illustrate the aneurysms with all the mentioned structures. In summary, our main contributions are:

- We present a design study for the visualization and exploration of internal blood flow and wall thickness that is based on fruitful discussions with medical experts and their hand-drawn images.

- We introduce novel illustrative visualization techniques for animated pathlines and the surrounding vessel wall thickness in a single, integrated, and interactive view.

- We integrate collaborative tools to improve the exchange of information. 
- We report on a qualitative evaluation that revealed the advantages of our developed techniques and framework and we compared our visualization techniques with state-of-the-art methods.

\section{Related Work}

Publications related to our submission comprise, in particular, the visualization of embedded structures, the visualization of blood flow, and the visualization and exploration of aneurysms.

VisuALIZATION OF EMBEDDED STRUCTURES. Diepstraten et al. [7] proposed different cutaway approaches to achieve interactive frame rates and Viola et al. [43] presented a view-dependent cutaway surface generation. In these methods, each part of the volumetric data has an object importance. Whenever unimportant structures occlude more important ones, an insight (i. e., a cutaway) is generated to ensure the visibility of important structures. Later, Liang et al. [25] introduced an approach to interactively cut a hole in a surface to reveal important structures behind it. As we use animated blood flow, we need an automatic approach to cut away a surface. Li et al. [24] also proposed exploration techniques by interactively defining target structures which their system uses to generate a cutaway illustration. Burns and Finkelstein [4] introduced an adaptive cutaway approach to reach real-time performance which is also applicable to dynamic objects-useful for our goal of visualizing blood flow. An interactive framework to create cutaways based on sketching input was introduced by Knödel et al. [21]. This framework, however, requires elaborate user interaction, which makes it too complex for medical researchers. Sigg et al. [38] proposed an approach to optimize the visibility of specific target objects. They use a view-dependent objective function to approximate the optimal solution of positioning cutaway objects. Lidal et al. [26] used predefined objects to generate a cutaway surface for encoding context information on it to distinguish layers of geological models. For our medical scenario, however, predefined objects may be too large such that the vessel morphology is cut away. Pindat et al. [33] introduced Gimlenses, a visualization technique for the interactive exploration and navigation through complex $3 \mathrm{D}$ models.

VISUALIZATION OF BLOOD FLOW. Mattausch et al. [28] presented different approaches for the interactive exploration of flow. With this approach, flow features can interactively be analyzed by mapping them to different visual attributes, e. g., width and opacity. Gasteiger et al. [14] introduced the FlowLens, an interactive visualization tool for the exploration of data on and inside a vessel. It can be used to explore the interior blood by showing the animated pathlines. However, with this approach only blood flow in a certain area-defined by the lenscan be observed. Thus, it is difficult to follow the flow outside the lens. Neugebauer et al. [32] proposed an approach for the qualitative exploration of near-wall blood flow. They used several 2D widgets to simplify the underlying streamlines at different positions on the surface. Van Pelt et al. [40] introduced a framework for the interactive exploration of $4 \mathrm{D}$ phase-contrast magnetic resonance imaging (PC MRI) data that represents cardiac blood flow. In this framework, crosssections can interactively be translated along the vessel and serve as seeding points for the pathlines. For the interactive virtual probing of 4D blood flow, van Pelt et al. [41] introduced another framework to position a probe semi-automatically. This tool focuses on the blood flow and does not visualize the vessel morphology. Born et al. [3] used illustrative visualization methods to provide a simplified blood flow representation. The main aspect of their approach is to simplify the blood flow without representing the vessel morphology. An overview on the visualization of simulated and measured flow data can be found in the summaries by Preim and Botha [34] and Vilanova et al. [42].

VISUALIZATION AND EXPLORATION OF ANEURYSMS. Based on contrast-enhanced computed tomography (CT) data, Higuera et al. [18] introduced the automatic adjustment of bidimensional transfer functions, using measured intensities and gradient magnitudes to visualize aneurysms. Neugebauer et al. [31] employed a 2D map display for the exploration of the cerebral aneurysm. They used a scalar field to decode information on the surface. They then visualized the surface as a 3D model as well as a flattened map surrounding the surface. While this approach enables users to explore the entire scalar field of the surface from a single viewpoint, no blood flow information is provided. Gasteiger et al. [15] visualize aneurysm surfaces based on Fresnel shading, which allows a simultaneous display of vessel morphology and blood flow. However, they shade the vessel parts which face away from the view direction more opaquely, thus occluding the interior blood flow information. Lawonn et al. [22] proposed a novel surface visualization based on suggestive contours. This shading technique distinguishes between convex and concave regions according to the viewpoint. Glaßer et al. [16] presented a framework for the visual exploration of vessels with wall thickness and hemodynamic information. Here, they visualized the wall thickness but a visual representation of the interior blood flow was missing.

None of these approaches, however, provide a combined visualization of embedded structures and the interior dynamic blood flow. We thus integrated different visualization approaches into our framework, combining the advantages of this prior work for vessel visualization. Moreover, our work adds a dynamic view of the internal blood flow.

\section{Medical Background}

We start by briefly summarizing the medical background and the image data acquisition for evaluating cerebral aneurysms. The rupture of a cerebral aneurysm can lead to a subarachnoid hemorrhage where blood flows in the ventricular system. This results in a high mortality rate of $45 \%-75 \%$ [35]. Therefore, research is essential to improve treatment options. The evolution and initiation of the disease comprise different factors such as genetics, hemodynamics, vessel morphology, inflammation, and further factors that are not well understood yet $[2,11]$. According to recent studies $[6,27]$, domain experts reported certain flow patterns to be important indicators for the initiation and outcome of a cerebrovascular disease. In particular, they observed a correlation between complex and instable blood flow patterns and the aneurysm's rupture risk. An effective visualization is thus necessary to allow medical researchers to assess the vessel hemodynamics. The needed multi-dimensional data can be derived from computational fluid dynamic (CFD) simulations or measured using 4D PC MRI with ultra-high field scanners (7 Tesla and beyond).

For hemodynamic analysis, neurosurgeons reported that an aneurysm's rupture risk depends on its wall thickness [19]. While a noninvasive in-vivo imaging technique to acquire the cerebral aneurysms' vessel wall is still missing, intravascular imaging such as intravascular ultrasound (IVUS) is currently employed in cardiology to depict the vessel lumen and wall morphology. Because it is not yet certified for use in the cerebral vasculature, current clinical research focuses on transferring intravascular imaging from cardiology to neuroradiology.

In this paper, we use a real-world IVUS dataset with measured wall thickness and simulated blood flow (hemodynamic dataset). In addition, we also use several aneurysm datasets. For this, the internal vessel geometry was measured and the blood flow was simulated in this geometry. Afterwards, the wall thickness was artificially generated to approve the functionality of our approach on several datasets. Our framework allows for both medical analyses: the evaluation of internal blood flow as well as the wall thickness.

We employ an IVUS dataset that was generated by measuring the wall thicknesses in an artificially created aneurysm in a porcine renal artery using IVUS (see Glaßer et al. [16] for more details). Hence, a branching artery was dissected and the shorter branch was artificially closed to provide a saccular aneurysm. Then, the IVUS dataset was acquired by probing along the vessel with the ultrasound probe at a constant velocity. The resulting stack of 2D grayscale images was used to generate a 3D surface and to extract the wall thickness.

The geometry of aneurysm datasets was derived from computed tomography angiography (CTA), magnetic resonance angiography (MRA), and 3D rotational angiography. The high vessel-to-tissue contrast in all these modalities facilitates a vessel segmentation using a threshold-based segmentation followed by a connected component analysis. By applying marching cubes to the segmented image data, a 3D surface was generated. In the preparation of a geometric model for CFD simulation, it is necessary to manually correct artifacts because these can lead to erroneously merging branches (details can be found in 

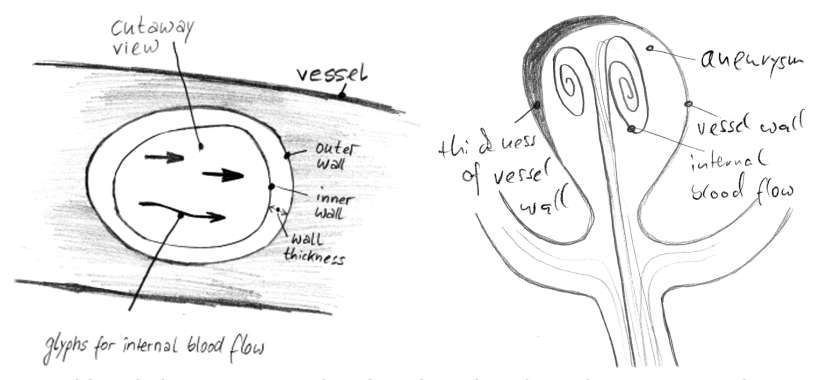

Fig. 2. Hand-drawn example sketches by domain experts who were asked for a cutaway view providing internal blood flow as well as a simultaneous visualization of the wall thickness.

$[13,30])$. To use these datasets, we manually smoothed their meshes by applying the technique of Mönch et al. [29] and then extruded the inner vessel wall to the outside to be able to visualize the wall thickness.

For both of the previously described dataset types, a separate simulated hemodynamic dataset is necessary to represent the blood flow. Using the generated or obtained 3D models, we determined a tetrahedral grid using Netgen (see Schöberl [37]). Based on this grid, we applied a CFD simulation to approximate the interior blood flow. Blood is considered to be a Newtonian fluid, so we used the Navier-Stokes equation in the simulation (for details see Cebral et al. [5]). Based on this flow dataset, we extracted the pathlines using an adaptive Runge-Kutta scheme [39]. We refer to the thesis of Gasteiger [13] for a comprehensive overview including visualization and exploration techniques.

\section{Requirement Analysis}

For developing a visualization framework that is able to depict the wall thickness with animated blood flow several requirements need to be fulfilled. Our approach is based on discussions with four domain experts: two physicians and two CFD engineers who work on hemodynamic analysis. We asked them to produce hand-drawn images, indicating how they would imagine a visualization that illustrates both the wall thickness and the internal blood flow, see the examples in Fig. 2. We discussed these drawings with them and asked them to state the most important quantities that need to be visualized. As the surface morphology and the flow behavior influence each other, the experts stated that they are interested in an integrated view such that both modalities are shown. They also commented that strong local changes of the vessel wall thickness are important. Moreover, they stated that the vessel wall at irregular shapes, e. g., blebs at daughter aneurysms, is essential. The vessel wall's changes indicate remodeling due to plaques and are thus essential to assess the risk of rupture. Furthermore, they mentioned that the flow behavior might be an important aspect as it may initiate or evolve a cerebrovascular disease. Therefore, the vessel morphology is an important requirement for the visualization (see also [15]). Furthermore, the experts commented that they need to know the behavior of the flow near the wall. They stated that near-wall flow may lead to pathological vessel dilation and, therefore, needs to be depicted for further analysis (see also $[12,17]$ ). Based on these discussions, drawings, and our analysis of the state of the art, we now summarize the main requirements for a visual exploration of vessel characteristics (i. e., wall thickness with internal blood flow) as follows:

Req. 1: Vessel view with wall thickness. We need an overview of the aneurysm, comprising the vessel morphology as well as wall thickness information. For the thickness, the domain experts want to be able to set up different thresholds during exploration. The experts stated that they prefer discrete colors on the vessel, in contrast to using a continuous color encoding. This facilitates a semi-quantification for the detection of dangerous regions, i. e., at thin vessel regions.

Req. 2: Visibility of flow. The blood flow needs to be visible during the whole animation. Experts can then trace the flow without encountering occlusion problems, and without the need to interact.

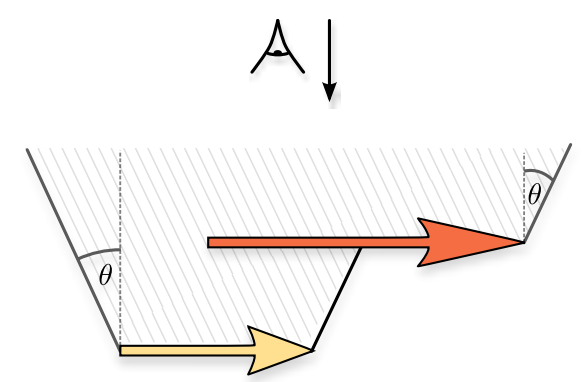

Fig. 3. Arrows of the flown seen from the side (i. e., the viewpoint is on the top). The opening angle $\theta$ defines the (gray) cutaway surface.

Req. 3: Revealing near-wall flow. During the exploration, the experts also need to understand the flow's distance to the vessel to distinguish between near- from far-wall flow: Near-wall flow may lead to a pathological vessel dilation, which increases the risk of a rupture. To ensure that these flow regions are passed by the pathlines the seeding of the flow is also very important—but this is not the focus of this paper.

Req. 4: Flow analysis at thin regions. The analysis of the blood flow behavior is essential for thin regions. Experts thus state that they first analyze the flow at thin regions before observing the pathlines in general. Further analysis tools are hence required that permit them to focus on the flow at different regions while also showing a contextual overview of the dataset.

Req. 5: Understanding the vessel morphology. For distant or overlapping vessel parts it is important to provide depth cues. These support users in understanding the vessel morphology and improve the perception of depth and the spatial layout of the vessel.

Req. 6: Collaboration tools. For collaboration purposes it should also be possible to set landmarks with different animation steps. An external researcher should be able to analyze the regions at the marked landmarks and discuss them with other colleagues.

\section{Basic Visualization Algorithms}

Based on these guidelines we set out to create an integrated representation of both the blood flow as focus and the vessel geometry as context. To meet Req. 2, we need an algorithm that ensures the visibility of the flow. We thus require real-time cutaway surfaces applied on the pathlines that guarantee an insight into the vessel to observe the animated blood flow. Therefore, we employ the cutaway technique by Burns and Finkelstein [4] which uses the algorithm by Rong and Tan [36]. Therefore, we summarize the concepts of both approaches next and demonstrate how we apply them to animated pathlines.

\subsection{Cutaway Technique by Burns \& Finkelstein [4]}

We employ a technique introduced by Burns \& Finkelstein [4] which is based on the depth image of the current scene and Rong and Tan's [36] jump flooding algorithm. Thus, we only use the depth image of the objects of interest - in our case the pathlines - to create the cutaway. Initially, we set the depth image to the following: if a pixel $p$ contains the object of interest $R$, we set its depth value to the pixel's computed $z$-value. Otherwise, it is assigned to 0 : Next we create a conic object around the objects of interest $R$ with an opening angle $\theta$, see Fig. 3 . We thus use a cutaway surface function $C$ :

$$
C(p)=\max _{q \in R}\left(q_{z}-m \cdot\left\|q_{x y}-p_{x y}\right\|\right),
$$

where $R$ contains the objects of interest, $m$ encodes the slope with $m=\tan ^{-1} \theta$, and $p_{x y}$ is the pixel's current 2D location.

\subsection{Jump Flooding Algorithm by Rong \& Tan [36]}

The jump flooding algorithm is used to determine the cutaway surface function $C$, recall Eq. 1, with real-time performance. Given an image of size $n \times n$, a standard flood fill algorithm would pass the content of a pixel to its 8 neighbor points. This algorithm needs at most $n$ 

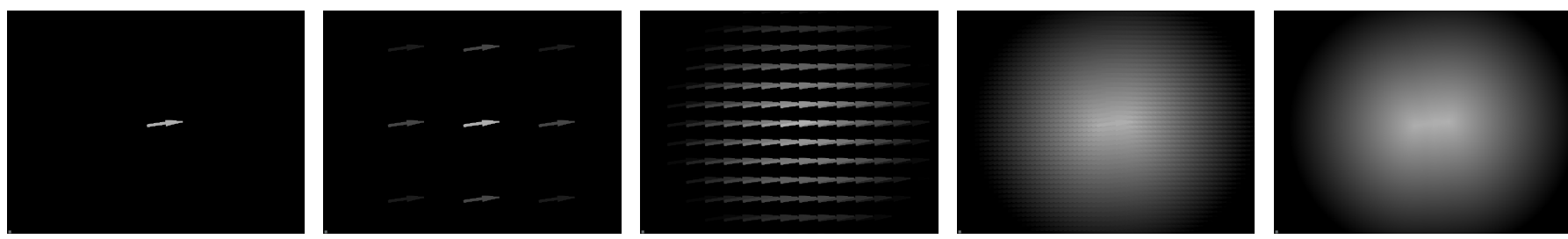

Fig. 4. The jump flood algorithm on a single arrow glyph with $i=\{0,2,4,6,8\}$ iterations. First, only the depth image of the arrow is generated. Then the jump flood algorithm is applied and a depth image is generated that can be used for the cutaway technique.

iterations to pass the content through the whole image.Contrarily, the jump flooding algorithm needs at most $\log n$ iterations. It changes the step size at every iteration $i$ to $k=n / 2^{i}$.At each iteration, a pixel at $(x, y)$ thus passes its content to its eight neighbors at $\left(x+l_{1}, y+l_{2}\right)$ with $l_{1}, l_{2} \in\{-k, 0, k\}$. We describe further refinements and details in Sect. 6.

\subsection{Pathline Animation}

Inspired by Everts et al.'s [9, 10] illustrative line styles, we use arrow glyphs to represent the pathlines. In addition, we encode line predicates, e.g., velocity or distance to the surface by color. The pathlines consist of points $\mathbf{x}$, each of which is assigned to two scalar fields: the integration time $t(\mathbf{x})$ and the distance to the surface $d(\mathbf{x})$. The integration time refers to the time at which a specific point is reached during the animation. We also set $T_{\max }=\max _{\mathbf{x}} t(\mathbf{x})$ as the global maximum integration time of all pathlines. During the rendering process, we know the current time $t_{c u r}$ with $t_{c u r} \in\left[0, T_{\max }\right]$. We draw the respective part of the pathline whenever the absolute value of the difference of the current time $t_{c u r}$ and the integration time $t(\mathbf{x})$ of a specific point $\mathbf{x}$ is smaller than the user-defined threshold width. This approach visually emphasizes faster pathline parts compared to other (slower) parts: the faster a section of the pathline is, the wider this part is drawn. Color assignment and glyph representation details are explained in Sect. 6.

\subsection{Centerline Extraction for Slice View Analysis}

We also provide a slice view to facilitate a detailed analysis of the wall thickness. Users can slide along the centerline of the vessel (determined using the approach by Antiga et al. [1]) using a cutting plane, giving them two views of the wall geometry. First, the intersection is displayed on the cutting plane itself, providing an initial orientation in the context of the integrated visualization. Second, a separate $2 \mathrm{D}$ view shows an orthographic projection to give users an undistorted view of the vessel geometry and the wall thickness.

\section{Visualization Design for integrated Depiction of Vessel MORPHOLOGY AND BLOOd FLOW}

In this section we outline the implementation details and explain how we fulfill the requirements derived in Sect. 4. First, we address the pathline glyph visualization. Then, we describe the realization of the jump flooding algorithm and finally we introduce the slice view.

\subsection{Pathline Visualization}

We use a novel glyph representation of the pathlines that emphasizes near-wall flow (Req. 3). The pathline visualization is based on viewaligned triangle strips. For these strips we define a width $\omega$ to extend the lines to become quads. For every adjacent point pair $\mathbf{x}_{i}, \mathbf{x}_{i+1}$ on the pathline, we determine the tangent vector $\mathbf{t}$ by subtracting the point and normalizing the resulting vector: $\hat{\mathbf{t}}:=\frac{\mathbf{x}_{i+1}-\mathbf{x}_{i}}{\left\|\mathbf{x}_{i+1}-\mathbf{x}_{i}\right\|}$. We determine the direction of the quad's extent by computing the cross-product of the normalized view direction vector $\hat{\mathbf{v}}$ with $\hat{\mathbf{t}}$. With each incoming adjacent point pair $\mathbf{x}_{i}, \mathbf{x}_{i+1}$, we pass four points to the geometry shader:

$$
\begin{array}{ll}
\mathbf{x}_{\text {out } 1}=\mathbf{x}_{i}+\omega \cdot \hat{\mathbf{t}} \times \hat{\mathbf{v}}, & \mathbf{x}_{\text {out } 2}=\mathbf{x}_{i}-\omega \cdot \hat{\mathbf{t}} \times \hat{\mathbf{v}}, \\
\mathbf{x}_{\text {out } 3}=\mathbf{x}_{i+1}+\omega \cdot \hat{\mathbf{t}} \times \hat{\mathbf{v}}, & \mathbf{x}_{\text {out } 4}=\mathbf{x}_{i+1}-\omega \cdot \hat{\mathbf{t}} \times \hat{\mathbf{v}} .
\end{array}
$$

This scheme constructs a view-aligned quad. Furthermore, we also pass texture coordinates to the fragment shader so that we can draw glyphs along the pathline. Here, we only need the width encoded as a parameter, ranging from $[-1,1]$. Then, we use the texture coordinates to create the arrow glyph. For this, the domain expert can set two different thresholds $f_{1}, f_{2}$ to distinguish between near-wall and far-wall flow. Here, the values are set such that $f_{\min } \leq f_{1} \leq f_{2} \leq f_{\max }$ holds, where $f_{\min }$ is the minimal distance from the pathline to the vessel and $f_{\max }$ correspond to the maximum distance. The expert can thus set different regions $R 1, R 2, R 3$ based on $f_{1}, f_{2}$. The distance $f$ is in region $R 1$ if $f \in\left[f_{\min }, f_{1}\right]$, in $R 2$ if $f \in\left(f_{1}, f_{2}\right)$, and in $R 3$ if $f \in\left[f_{2}, f_{\max }\right]$. Initially, the values are set as $f_{1}:=0.1 \cdot\left(f_{\max }-f_{\min }\right)+f_{\min }$; i. e., $10 \%$ of the nearest flow is set for the first value and $50 \%$ is set for the second value: $f_{2}:=0.5 \cdot\left(f_{\max }+f_{\min }\right)$. During the animation, we draw glyphs that are in the range $\left[t_{\text {cur }}-\frac{\text { width }}{2}, t_{\text {cur }}+\frac{\text { width }}{2}\right]$ and, thus, set $u \in[0,1]$.

The glyph shape for the different regions $R 1, R 2, R 3$ is defined as follows. For $R 1$ with $f \in\left[f_{\min }, f_{1}\right]$ :

$$
v(f, u):= \begin{cases}0.25, & \text { if } u \in[0,0.5) \\ 2 \cdot(-u+0.9), & \text { if } u \in[0.5,0.9) \\ 0, & \text { if } u \in[0.9,1] .\end{cases}
$$

For $R 2$ with $f \in\left(f_{1}, f_{2}\right)$ :

$$
v(f, u):= \begin{cases}\frac{f-f_{1}}{f_{2}-f_{1}} \cdot(-0.125)+0.25, & \text { if } u \in[0,0.5) \\ 2 \cdot(-u+0.9) \cdot \frac{f_{2}-f}{f_{2}-f_{1}}, & \text { if } u \in[0.5,0.9) \\ 0, & \text { if } u \in[0.9,1] .\end{cases}
$$

For $R 3$ with $f \in\left[f_{2}, f_{\max }\right]$ :

$$
v(f, u):=0.125, \quad \text { for all } u .
$$

Here, $f$ is the distance of the pathline's fragment to the vessel. In $R 2$, we also change the width of the contour of the pathline slightly from 0 to 0.1 with $0.1 \cdot \frac{f-f_{2}}{f_{1}-f_{2}}$ illustrated in black. In $R 1$, we change the offset slightly from 0.1 to 0.2 , while a white contour is also generated around the arrow from 0 to 0.1 . Fig. 5 illustrates this change. Furthermore, we add a small depth offset for the halo to obtain results similar to the depth-dependent halos by Everts et al. [8]. Finally, we set the glyph color according to its second line predicate, e. g., the distance to the wall. This color is defined by determining the difference of the vessels' back and front face depth and the difference of the pathlines' depth with the front face depth. This creates an encoding that shows whether the pathline is near the current vessel in view direction or not-recall Fig. 1 and see Fig. 11. Here, we used a color scale ranging from dark red to dark blue with white in between. Thus, depending on the pathline glyph, the expert can differentiate between near-wall flow from far-wall flow, and furthermore he can distinguish if the flow is near the front face of the inner vessel or the back face of the inner vessel, see Fig. 6. Therefore, the changing style of the pathline glyphs as well as the color coding allows to detect near-wall flow and hence fulfills Req. 3.

\subsection{Jump Flooding Details}

To determine the cutaway surface function $C$ (see Eq. 1) we use a compute shader and the OpenGL extension SHADER_IMAGE_LOAD_STORE. We apply this technique to the pathlines to increase the visibility of the flow (recall Req. 2). First, we determine the depth image of the objects of interest and load it into the COMPUTE_SHADER using frame buffer objects (FBOs). Then, we apply the jump flooding algorithm and store the 


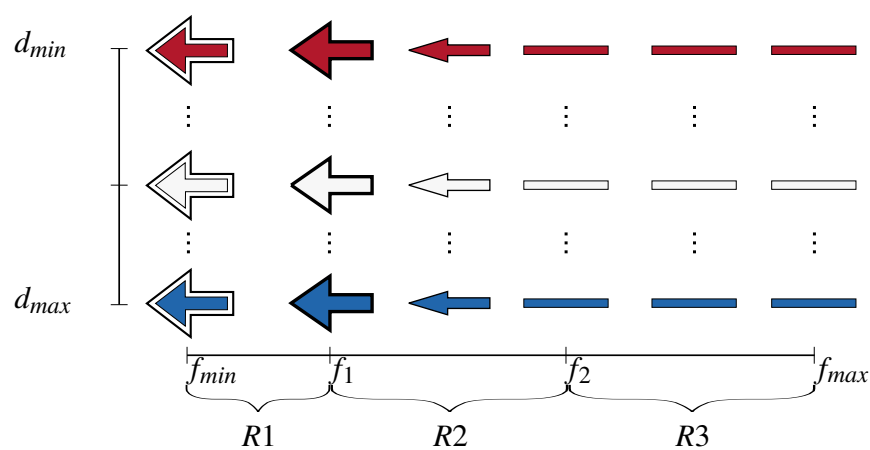

Fig. 5. Illustration of the continuous change of the pathline glyphs from far-wall to near-flow flow. R1, R2, and R3 represent the different regions.

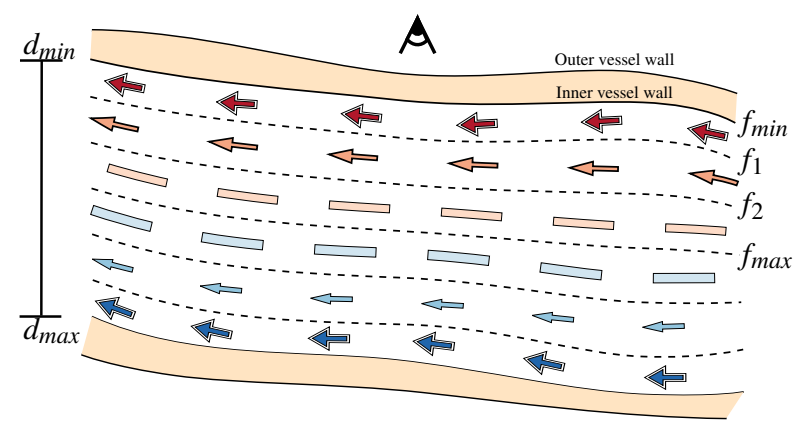

Fig. 6. Ilustration of the applied pathline glyphs with color coding. Nearwall flow is represented as arrows with a white contour. The flow near the camera appears dark red, whereas near-wall flow very far to the backside of the vessel is encoded as dark blue.

values in the RGBA channels of a cutaway texture using the OpenGL function imagestore(). The RG channels store the $2 \mathrm{D}$ position $(x, y)$ of the pixel that maximizes Eq. 1. These coordinates change during the iterative process. Initially, these values are empty. The B channel carries the depth of the neighbor resulting from the algorithm described in Sect. 5.2, which is initialized with their own depth. The A channel stores the $C(p)$ value. In the first iteration $(i=1)$, the step length is $k=n / 2$. Thus, for a given pixel $p$ we consider its (maximum) eight neighbors $q^{1}, \ldots, q^{8}$. Then, the cutaway surface function is determined for $p$ and its neighbors:

$$
C(p)=\max _{j \in\{1, \ldots, 8\}}\left(q_{z}^{j}-m \cdot\left\|q_{x y}^{j}-p_{x y}\right\|\right) .
$$

Let us assume the pixel $q^{j}$ maximizes $C(p)$. Afterwards, $C(p)$ is compared to $p_{w}$ (which stores the depth of the best neighbor that maximizes Eq. 1 from a previous iteration step). We keep its value if the current depth $p_{w}$ is greater than $C(p)$. Otherwise, we set $p_{w}:=C(p)$, $p_{x y}:=q_{x y}^{j}$, and $p_{z}:=q_{z}^{j}$, where $x y z w$ is encoded in the RGBA channel. Therefore, the pixel $p$ stores the information of the depth of the cutaway surface, and furthermore it records the position and the depth of the neighbor that maximizes $C$. The algorithm terminates after $\log n$ iterations. The iteration process is shown in Fig. 4.

\subsection{Cutaway Illustration}

The cutaway ensures the visibility of the flow (related to Req. 2). It is visualized using frame buffer objects (FBOs). The complete scene consists of images that are depth-sorted in a dedicated shader which uses the depth of the cutaway surface to determine if the outer or inner vessel is drawn. We draw a thin white border around the cutaway to highlight the flow in the vessel; recall Fig. 1. During the animation and interaction, the user is thus able to focus on the flow as it is emphasized.
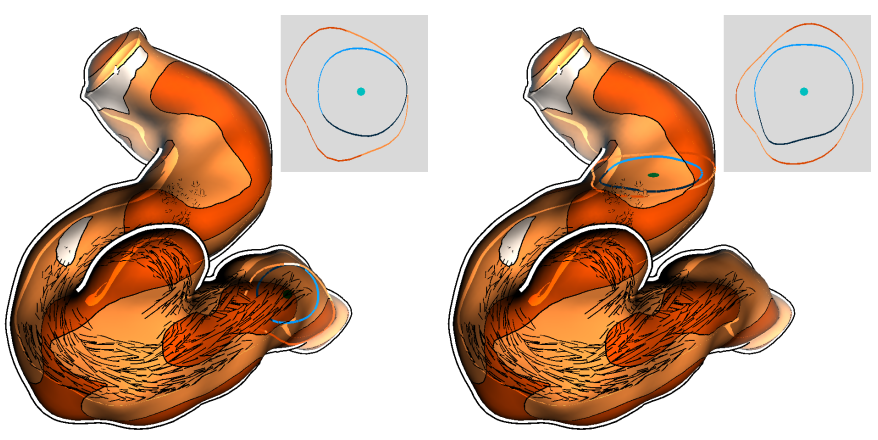

Fig. 7. The slice view facilitates the thickness estimation. Here, two different positions of the slice view are represented.
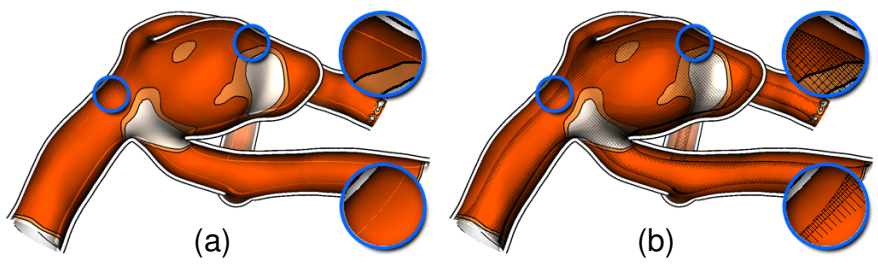

Fig. 8. Different illustration techniques that visualize the inner vessel part. In (a) the contour of the vessel is illustrated, whereas in (b) it is illustrated using image-based hatch strokes.

\subsection{Slice View}

As described in Sect. 5.4, the centerline provides a natural choice for the slice view navigation, which is therefore related to Req. 1. The centerline consists of consecutive points $\mathbf{p}_{i}, \mathbf{p}_{i+1}$ and the tangent $\hat{\mathbf{t}}_{i}=\frac{\mathbf{p}_{i+1}-\mathbf{p}_{i}}{\left\|\mathbf{p}_{i+1}-\mathbf{p}_{i}\right\|}$ is used as the normal of the cutting plane. Having a user-defined parameter $s$ for the slice view in the range $[0,1]$, we first determine the exact position $\mathbf{q}$ of the plane with:

$$
\begin{aligned}
& j=\lfloor n \cdot s\rfloor \\
& \mathbf{q}=\mathbf{p}_{j}+(n \cdot s-j) \cdot \hat{\mathbf{t}}_{j} .
\end{aligned}
$$

This plane divides the space into two half spaces. We test for every triangle of the vessel geometry if two of its three points lie in a different half space than the third one. In this case, we determine the intersection points of the corresponding edges with the plane and connect both points. This geometrical intersection is represented in the form of lines. We then position the camera on the tangent vector and add this view as an additional 2D widget in our framework, see Fig. 7.

\subsection{Vessel Illustration}

In the following, we describe our vessel illustration with the wall thickness visualization to satisfy Req. 1 . The vessel visualization is divided in two parts: (a) the visualization of the outer vessel wall and (b) the visualization of the inner vessel wall. For the inner vessel wall, the user can choose between two different rendering styles. The first version is an illustrative margin around the contour (Fig. 8(a)). This visual encoding provides contextual information of the inner structure. The alternative is the use of image-based hatch strokes, which are generated at the contour margin of the surface (Fig. 8(b)). The hatching is parameterized by the dot product between the view direction and the normal, using more strokes for smaller dot products (i.e., closer to the actual silhouette). Fig. 8 directly compares the two versions and Fig. 13(b) shows another example of the hatching technique. The backfaces of the inner vessel are also drawn at regions only where the cutaway surface provides a view into the vessel. The outer vessel is first visualized with a scalar field representing the thickness, ranging from white to brown, see Fig. 9. For the thickness computation per vertex $\mathbf{v}$ of the outer vessel, we iterate over all vertices of the inner vessel wall and determine the Euclidean distance to $\mathbf{v}$. The smallest value is assigned to $\mathbf{v}$. The user can select the thickness values in order to discretize the thickness information. 

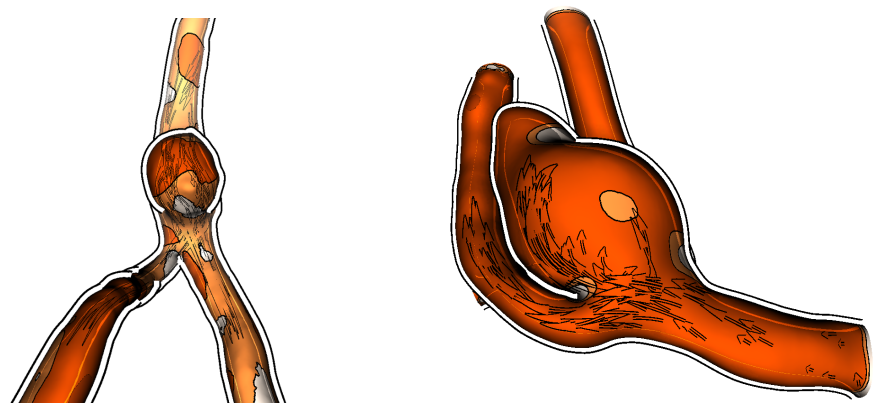

Fig. 10. Different views to analyze the surface where pathlines serve as context information.

Fig. 9. Four colors representing the wall thickness from low to high. The user is free to adjust this number of discretized colors to his/her needs.

In addition, the opacity of the discretized values can be set as well. This supports the domain expert to investigate the flow at specific regions. Here, the domain expert can set the opacity at thin regions to 0 and can thus analyze the flow at these regions only. As default values, we set $0,0.2$, 0.4 , and 0.8 with regard to the regions ranging from the thinnest to the thickest region. This allows to explore the near-wall flow in these regions and, therefore, satisfies Req. 4 . In addition, we implemented screen-space ambient occlusion [20] to improve the perception of the vessel through depth cueing following Req. 5.

\subsection{Depth Attenuation}

To increase depth perception, we add an atmospheric attenuation to objects that are far away from the camera according to Req. 5. For this purpose, we determine the depth of the current fragment $z_{f}$. We normalize it with the minimum $z_{\text {near }}$ and maximum $z_{\text {far }}$ depth values in eye space: $z_{n}=\frac{z_{f}-z_{\text {near }}}{z_{\text {far }}-z_{\text {near }}}$. If $z_{n}$ exceeds 0.45 , we add $5 \cdot\left(z_{n}-0.45\right) \cdot(1,1,1)$ as an RGB color to the current fragment to slightly increase its brightness. We set the threshold to 0.45 because a lower value would result in parts close to the camera becoming too bright, and a greater value would not affect the depth attenuation much.

\subsection{Framework}

This section describes the framework of our visualization tool. The GUI is divided in two parts: the standard GUI with all important settings and the playbar. The standard GUI provides pathline-related and vessel-related features. For the pathline settings, the user can adjust the animation speed, the pathline length, and the opening angle $\theta$ of the cutaway surface. The user can also choose between the novel glyphs and normal arrows as pathline representation. Furthermore, if the user wants to analyze the vessel wall surface, the pathline visualization can be turned off. The cutaway can also be deactivated to explore the surface with the pathlines as context objects, as shown in Fig. 10. This might be important as the expert needs the pathlines only as context information to explore patterns inside the vessel at thin regions.

The settings for the visualization comprise facilities to adjust wall thickness regions, to adjust distance parameters for the pathline glyphs and to highlight thin regions. Thin regions exhibit a higher risk of rupture, making these regions relevant for investigation as indicated in Req. 1 and Req. 3. Thus, the user can adjust a parameter such that those parts of the vessel are highlighted which fall below this thickness value. This approach facilitates a fast perception of potentially dangerous zones, even if the vessel includes many thin regions such that color coding may not be sufficient.

Req. 6 indicates that save and load facilities (for data, views, parameter settings, and animation) are needed to support the collaboration between medical experts. Furthermore, we included a feature that allows them to place landmarks on the surface. After the placement, a screenshot is taken and all important parameter settings and the camera properties are stored. Another domain expert can see the landmarks,
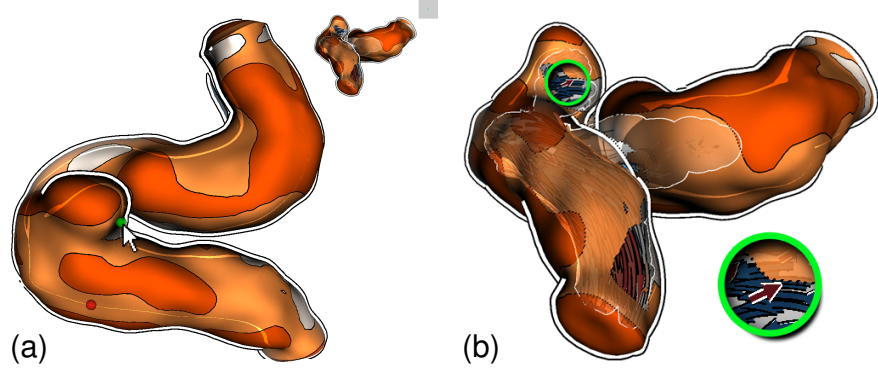

Fig. 11. A domain expert created landmarks, represented as red spheres. Another expert can select the landmark, resulting in a small preview (a). A click on it loads the dataset with the first domain expert's settings (b).

represented as red spheres, and can select them. A short preview is then shown in the top right corner as depicted in Fig. 11(a). A click on it loads the scene as shown in Fig. 11(b). In this case, the domain expert made a very important finding. She discovered that the flow is close to the vessel wall, in a region where the wall is very thin.

For the visual exploration of the pathlines, we created an interface similar to typical movie players. We thus provided standard buttons with their well-known functionality as well as a timeline to facilitate a fast navigation through the animation. Users can click on the timeline and the animation jumps to the corresponding keyframe. Furthermore, dragging the animation is possible for fast navigation through the simulation. Consecutive keyframes can also be shown by clicking on the previous or forward button.

\section{Comparison With RELATEd Approaches}

Closest to our visualization techniques is the work by Lawonn et al. [22] and Glaßer et al. [16]. Lawonn et al. [22] presented a shading technique that enhances the surface while near-wall blood flow is emphasized by a change of the surface color. While this approach highlights the surface regions where the blood flow is near the vessel wall, it does not visualize the wall thickness. Glaßer et al. [16], in contrast, focused on the wall thickness and used different visualization concepts to analyze dangerous regions based on the wall thickness, yet without showing the internal blood flow. We thus compare our visualization technique to these other two concepts.

\subsection{Near-Wall Visualization}

The vessel visualization by Lawonn et al. [22] is based on a suggestive contour measure to determine the shading (see Fig. 12(a)). Based on the surface's local shape (convex vs. concave) as well as on the distance of the pathlines to the vessel, they determine the rendering parameters (stored in a 2D lookup table). During animation, the changing flow leads to a highlighting of those vessel parts that are close to the blood flow. This visual encoding facilitates an analysis of surface regions that are close to fast blood flow. However, it only supports a qualitative estimation of the near-wall flow, as we demonstrate with a comparison of theirs with our approach in Fig. 12. The first close-up in Fig. 12(c) shows that our glyph-based approach reveals that the pathlines are close to the vessel wall - a fact that is invisible in the technique by Lawonn et al. The blue arrows in our visualization indicate that the pathlines are near the backside of the vessel. While the pathlines are also close to the wall in the visualization by Lawonn et al., this fact cannot be perceived due to the region highlighting. Furthermore, unlike our approach, important near-wall flow details may be overlooked in the visualization by Lawonn et al. if the view does not show the right vessel region. We demonstrate this effect in the second close-up in Fig. 12(c): only the red color around the contour indicates the near-wall flow, but for surface regions directly facing the view direction this effect is hard to perceive. With our technique the white outline around the arrow shows that the pathlines are close to the vessel. Moreover, the red pathlines show that it is close to the facing vessel part. 

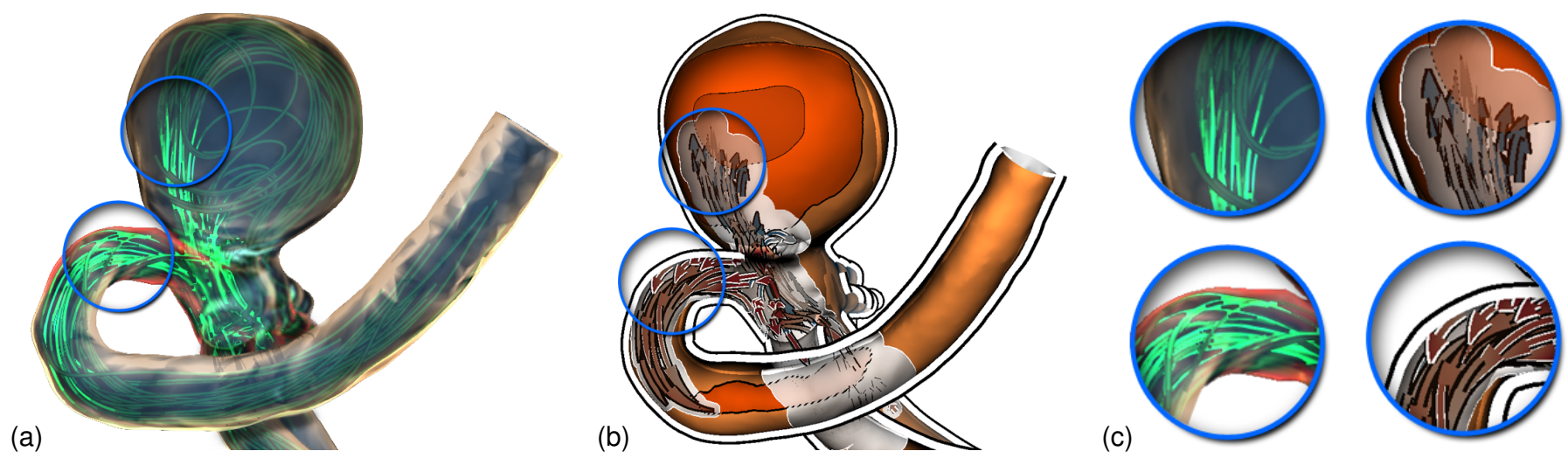

(c)
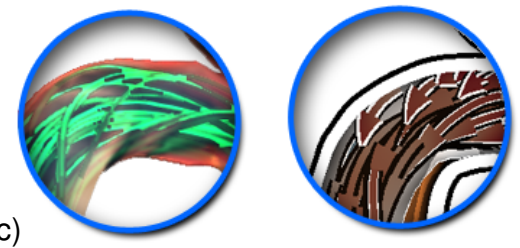

Fig. 12. Close-ups (c) of the visualization technique by Lawonn et al. [22] (a) and our new technique (b). Our pathline glyphs support the illustration of the near-wall flow better since the arrow glyphs with the white contour indicate near-wall flow. Furthermore, it can be better distinguished if the pathlines are near the front faces or the back faces of the vessel wall, here colored in red and blue, respectively.

(a)

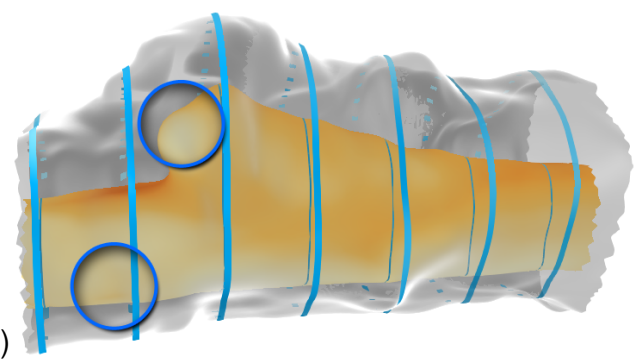

(b)

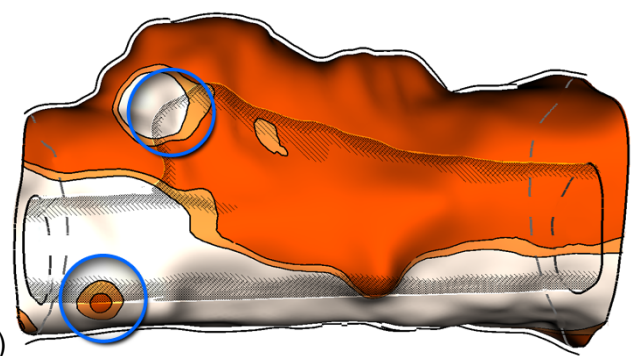

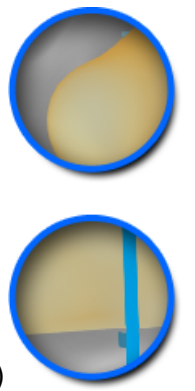

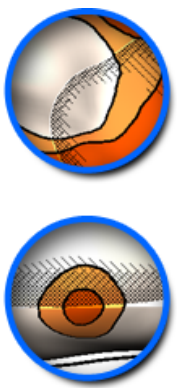

Fig. 13. Close-ups of the visualization technique by Glaßer et al. [16]: (a) and our new technique (b) with the IVUS dataset. Our wall thickness visualization facilitates the identification of small, thin regions, while the visualization of the inner vessel provides a contextual visualization.

\subsection{Wall Thickness Visualization}

Glaßer et al. [16] designed a framework for the analysis of the wall thickness. They used a slice view, distance ribbons, and an outer wall representation to visualize the thickness and to facilitate a qualitative analysis (Fig. 13(a)). None of these techniques, however, allows physicians to measure the thickness quantitatively. Fig. 13 directly compares our visualization technique with the method by Glaßer et al.; the two close-ups in Fig. 13(c) demonstrate the advantages of our wall thickness visualization. The first close-up illustrates a thin region, and both visualization techniques show the region well. However, with our discrete wall thickness representation we can immediately identify a dangerously thin region which can be overlooked in the continuous visualization by Glaßer et al. The related issue of identifying thick regions is shown in the second close-up in Fig. 13(c): the thick region can easily be missed with the approach by Glaßer et al.

\subsection{Summary}

Our technique allows physicians to analyze the wall thickness quantitatively while simultaneously representing the blood flow. By also depicting the pathlines with our glyph-based approach we facilitate a quantitative analysis of the flow near the vessel wall. Therefore, nearwall flow can be perceived even if the flow is near the backfaces of the vessel (i.e., far away from the viewer). Furthermore, thin regions independent of their size - can be perceived using our discrete visualization. In summary, compared to Lawonn et al., our technique is more appropriate to reveal near-wall flow (recall Req. 3). The method by Glaßer et al. allows a visual analysis, as brushing \& linking is applicable, but in comparison to our technique, our technique improves the vessel wall thickness representation (recall Req. 1). Our new visualization techniques combines the advantages of both approaches.

\section{Qualitative Evaluation}

Our design process comprised several iterations with the domain experts. We now describe the first feedback cycle for our initial prototype that did not yet comprise the complete functionality presented so far.
Six unpaid participants ( 1 female, 5 male; $26-45$ years old; median 35 years) took part in our study. Among them were the four domain experts that already helped us with defining our initial concept: two physicians (14 and 15 years of professional experience) and two CFD engineers involved in hemodynamic analysis (both 5 years of professional experience). To get additional independent feedback, we added two researchers with background in medical visualization.

We briefly presented our framework and described its features to the participants. Next, we encouraged them to explore the tool on their own, while we recorded their oral comments and their interaction with the framework. Finally, we asked them to fill out a questionnaire.

\subsection{Questionnaire}

The questionnaire consisted mainly of questions about medical aspects, our visualization, the framework design, and our parameter choice. Depending on the question, we asked for yes/no responses, ratings on a five-point Likert scale $(--,-, \circ,+,++)$, or open-ended comments.

Demographics/background. First, we collected the typical participant demographics: gender, age, and medical and/or technical experience. We also inquired about our participants' background in computer-assisted analysis of aneurysms and about their experience in analyzing simulated blood flow.

Medical questions. Next, we asked about the importance of the wall thickness for medical evaluation and about the importance of pathlines. We also asked about which elements are important for a diagnosis of cerebral aneurysms and how this is currently visually supported.

Visualization questions. We asked our participants to comment on the different wall thickness visualizations we provide. Specifically, we asked about the slice view and the highlighting of surface regions with walls thinner than a given threshold. Next, we asked whether the color coding of the pathlines is useful to highlight near-wall flow. We also asked if the glyph-based representation with varying length allows them to differentiate between slow and fast-moving flow. Then, we inquired whether the playbar facilitates an effective temporal data exploration. Finally, we asked if our cutaway visualization enables them to follow 
Individual colors for Likert score:

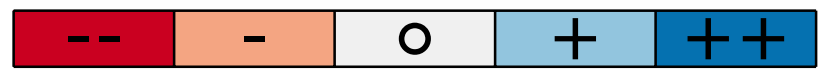

Medical questions:

Importance of wall thickness

Blood flow and wall thickness

Estimation of flow with glyphs

Visualization questions:

Slice View

Highlighting

Color coding of near-wall flow

Varying lengths of pathlines

Animated pathlines

Framework aspects:

Pathlines can be followed

Cutaway for pathlines analysis

Playbar interaction

Saving and loading the scene
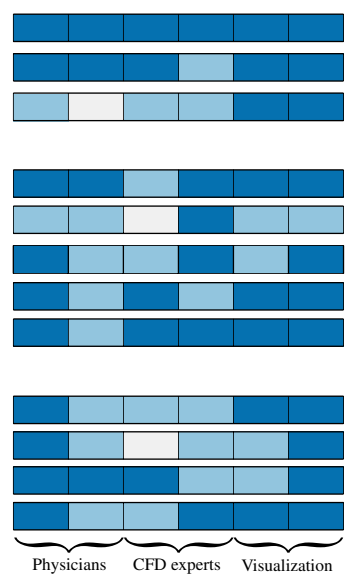

Fig. 14. The results of the evaluation with color-encoded Likert scores. Each box represents the answer of one participant: the two left-most boxes depict the physicians, the middle two boxes show the CFD experts, and the two right-most boxes represent the medical visualization experts.

the animated pathlines and if it is helpful for the analysis.

Framework aspects. Here we asked if the participants had any problems when exploring the vessel with internal blood flow. Furthermore, we inquired if the saving/loading options are useful for the analysis as well as for the collaboration with other medical experts. We also asked whether any problems occurred during the exploration and about potential suggestions for the future development of the technique. We then asked if the participants could imagine to use our framework for their research. Finally, we asked for any additional remarks.

\subsection{Results and Discussion}

The overall result of the empirical evaluation is shown in Fig. 14. No participant had any major problem with using our framework. After a short introduction, the participants could immediately use the tool. All stated that they would like to use the tool for the exploration of wall thickness and blood flow. In particular, the domain experts confirmed the high importance of analyzing the wall thickness for the rupture risk assessment of aneurysms. They also stated that the combination of wall thickness and blood flow visualization is important for further analysis of cerebrovascular diseases. Next, we discuss selected aspects and insights from our evaluation.

Cutaway. All participants stated that the cutaway is useful to follow the animated blood flow and that it provides a natural view into the vessel. One physician stated that this animation is confusing due to the constant change of the cutaway. Hence, pausing of the animation was required to stop this change. Nevertheless, he appreciated the use of the cutaway for analyzing the pathlines. The usefulness of being able to follow the pathlines and to analyze the interior flow behavior was rated by the physicians with ' ++ ' and ' + '.

Glyph-based pathlines. Most participants described the color coding of the pathlines as essential to be able to distinguish between near-wall and far-wall flow. All participants appreciated the length encoding of the glyphs to represent the velocity. One of them stated that it is very helpful for still images because this representation conveys that the flow is slower in the aneurysm compared to the flow in the vessels. Moreover, one can directly see the flow direction.

Wall thickness. The results show that participants preferred the slice view over the $3 \mathrm{D}$ view to understand the wall thickness. The reason may be that, in our first iteration, we used a continuous color coding for the thickness. The highlighting was also considered as essential to assess danger zones. Most participants first used the highlighting, later looking at slices for a more detailed analysis of the thickness.
Playbar. All participants found the playbar useful: they could explore all time steps and analyze the pathlines. Mostly, they first played the whole animation and then looked for specific points in time for further examination using the animation bar. Finally, the participants used the forward and backward buttons for further detailed analysis.

Application domains. We also asked our participants about application domains they could envision for the presented tool. Both physicians explained that our tool is useful for aneurysm risk assessment and for treatment planning. All participants also agreed that another important application field is research and education. Some participants mentioned that our technique may also be helpful for patient information and may be used to explore the predicted blood flow after different treatment options, such as coiling and stenting.

Performance. For all presented models (e. g., Fig. 1, 10, 15, and 16), we achieve a real-time performance of approximately $41 \mathrm{fps}$. We used a laptop with an Intel Core i5 with $2.6 \mathrm{GHz}, 8 \mathrm{~GB}$ RAM, and an nVidia GeForce $870 \mathrm{M}$ with $4 \mathrm{~GB}$. This performance test was conducted with a resolution of $1920 \times 1080$ and we used the half of the resolution (approx. $960 \times 540$ ) for the cutaway surface.

Improvements. As already indicated, based on this evaluation and concrete suggestions we added several additional visualization techniques and encodings. One participant stated that it might be difficult to distinguish those vessels that are near to the camera from other vessels which are further away. Therefore, we added the depth attenuation described in Sect. 6.6. Another participant wanted us to add features to only visualize the inner wall, to visualize the centerline, and to enlarge the slice view on a mouse-over. We added all these ideas and he liked the improvements. Then, we changed the wall thickness representation from a continuous color coding to a discrete color coding with parameters that can be set by the experts. Next, we also proposed the new glyph-based representation of the pathlines to better distinguish between near-wall and far-wall flow. Furthermore, we added colors to make it clear if the pathlines are near the front or the back faces. As suggested by our medical experts, many additional collaboration tools were added such as the ability to add, store, and retrieve landmarks. Finally, we added the hatch-based illustration of the inner vessel wall since one expert had asked for a more contextual visualization.

\section{EXPERT FEedBack}

To evaluate our improvements, we conducted another evaluation similar to a focus group [23]. Here, we gave the participants the opportunity to test our improved framework and to analyze its functionality. We then discussed our tool with three researchers with background in CFD simulation (males; 27, 30, and 35 years old) and one physician with an emphasis on surgery (female; 29 years old). We abbreviate the $\mathrm{CFD}$ researchers with $\mathrm{C} 1, \mathrm{C} 2$, and $\mathrm{C} 3$ and the physician with $\mathrm{P} 1$. The participants were asked to make positive as well as negative comments. The interviews took approximately 40 minutes.

First, all participants stated that the analysis of the wall thickness with the animated blood flow is a great extension to state-of-the-art visualization frameworks. Then, we asked them to share their impressions of the framework. P1 stated that it would be a nice feature to have animated camera paths to navigate around the vessel. C2 had the idea that it might be an interesting feature to activate multiple linked views. Then, one could activate different parameter settings like wall shear stress and the user can analyze different parameters simultaneously.

Furthermore, the physician wished to have the original image data in the slice view. The CFD experts also wanted to see the original data to estimate the uncertainty of the vessel reconstruction. In fact, the experts stated that our technique is applicable to both the analysis of hemodynamic information according to near-wall flow as well as wall thickness. Moreover, they stated that the glyph-based approach is very helpful to observe where the flow is close to the wall. Setting own thresholds makes it easy to explore the dataset. P1 explicitly emphasized the advantages of the slice view as certain regions can be observed. C3 mentioned that it would be nice to have dashed contour lines whenever the flow is occluded by a vessel. In addition, C3 stated that the color coding as well as the glyph representations with the white contour allows a fast perception of the near- and far-wall flow. 

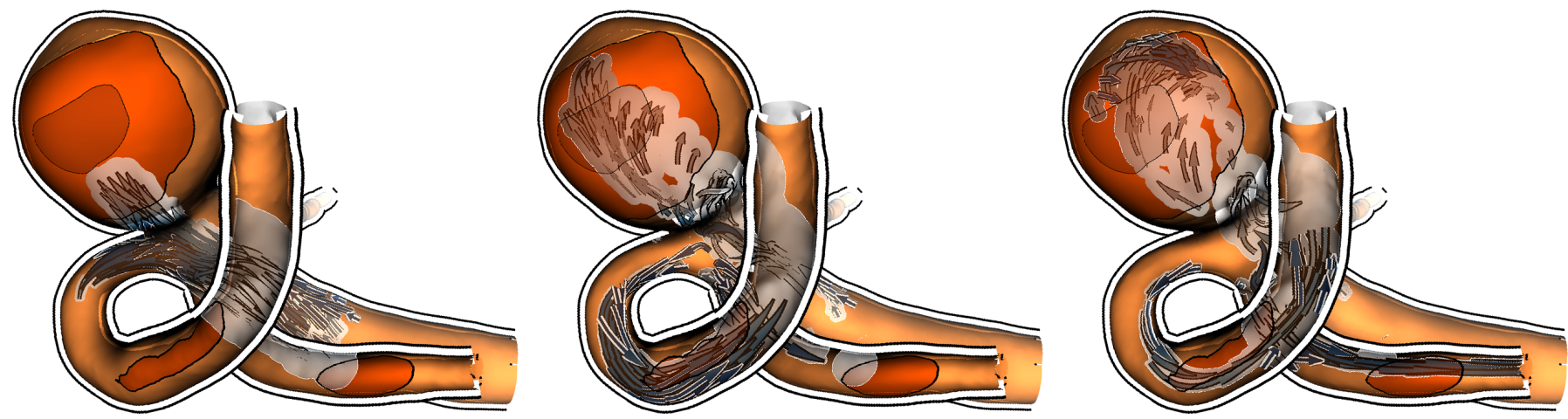

Fig. 15. Dynamic cutaway visualization of pathlines within the context of the vessels and aneurysm: Three steps within the cardiac cycle.

Furthermore, he said that the color coding makes it easy to distinguish between the near-wall flow that is close to the front or the back faces. The participants also pointed out that a main benefit of our approach is its placing of landmarks, which easily allows to collaborate with other experts. Here, $\mathrm{C} 3$ stated that it would be nice to include text at the landmarks. With this, the mouse-over not only shows a small preview, but a text is also shown, which states the specific observation made by the expert. This was also stated by $\mathrm{C} 2$ who said that the initials of the domain expert who placed the landmark should appear. This would be beneficial when several researchers work on the same dataset. Furthermore, he recommended to blur the white line indicating the cutaway surface which would make the lines less obtrusive. P1 stated that the color representation is a great benefit to easily distinguish between thin and thick regions. She also mentioned that the different glyphs make it easy to perceive near-wall flow and that the color coding helps to analyze the blood flow. In addition, P1 said that seeing a blue glyph with a white contour leads to a fast understanding where the pathline is located. In essence, P1 stated that dangerous regions can be easily identified and analyzed with the interior blood flow. Finally, the participants stated that a tool that includes our analysis of wall thickness and hemodynamics would be of great benefit to them.

\section{Conclusion ANd Future Work}

In this paper, we presented a design study comprising a visualization technique and an exploration tool for the analysis and exploration of vessels using an integrated wall thickness and internal blood flow representation. Some final results are shown in Fig. 15 and Fig. 16. Based on the requirements derived from consultations with medical experts and a literature analysis, we combine a number of known rendering and visualization techniques with several novel technical concepts. In particular, we contribute a technique for glyph-based visualization to represent data values along streamlines or pathlines. All these methods put together, we are able-for the first time-to present an integrated illustrative visualization of all essential elements for aneurysm assessment—in particular including a representation of a vessel's wall thickness. Our visualization framework thus allows users to analyze the pathlines using interactive animations and without occlusion issues. Furthermore, we integrated modern visualization methods to the surfaces and to the pathlines to depict further flow attributes such as stress forces and velocities. We make use of illustrative elements such as color coded arrow glyphs to represent the flow direction, velocity, and pathline distance even on still images.

Thanks to our GPU implementation we achieve real-time rendering speeds even on mid-class computers. We integrated our visualization technique into a data exploration framework that facilitates the exploration of the vessel data. To assess our results, we conducted a qualitative evaluation with our collaborating experts. This evaluation provided evidence that our overall approach is valid, confirmed many of our design choices, and provided several additional paths for improvement - some of which we realized, presented, and re-evaluated with further domain experts. We thus provide a comprehensive illustrative visualization approach for the analysis and exploration of aneurysms and their internal blood flow that is applicable in medical

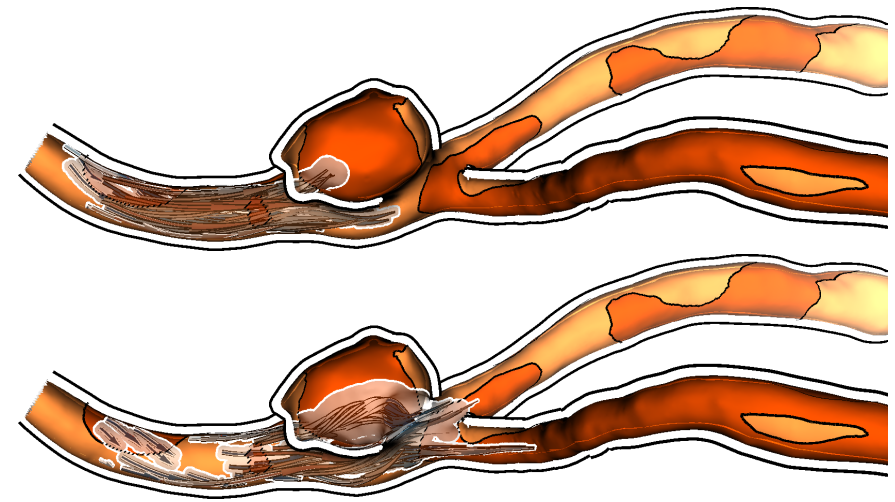

Fig. 16. Two subsequent time steps of the animated pathlines in a vessel.

research and other domains.

Although we evaluated our visualization technique with positive feedback, some issues still need to be improved. First, while the slice view is helpful to get an impression of the wall's thickness at specific points, the medical experts are also interested in the original image data which we are currently not showing. Furthermore, to analyze the wall thickness it might be helpful to include a ruler to measure the thickness on specific parts of the vessel. More interaction can be integrated by selecting a region to obtain different quantities, e. g., the average wall thickness or the wall shear stress. As soon as modern imaging technology is available, we would also like to visualize additional information on the inner vessel structure onto the cutaway surface such as calcified parts or plaque. This would allow medical experts to gain further insights into the vessel's structure. Finally, we would like to improve the CFD simulation to include biomechanic properties of the vessel such that dynamic changes of the vessels which could then also be included in the visualization tool.

Nonetheless, based on the described evaluations we consider our method as successful in filling the gap between visualizing wall thickness and illustrating hemodynamics with highlighted near-wall flow.

\section{ACKNOWLEDGEMENTS}

This work was partially funded by the BMBF (STIMULATE-OVGU: 13GW0095A). The authors like to thank Sylvia Glaßer and STIMULATE for providing us the IVUS dataset.

\section{REFERENCES}

[1] L. Antiga, M. Piccinelli, L. Botti, B. Ene-Iordache, A. Remuzzi, and D. A. Steinman. An image-based modeling framework for patient-specific computational hemodynamics. Medical \& Biological Engineering \& Computing, 46(11):1097-1112, 2008. doi> 10.1007/s11517-008-0420-1

[2] S. Appanaboyina, F. Mut, R. Löhner, C. Putman, and J. Cebral. Computational fluid dynamics of stented intracranial aneurysms using adaptive embedded unstructured grids. Numerical Methods in Fluids, 57(5):475493, 2008. doi > 10.1002/fld.1590 
[3] S. Born, M. Markl, M. Gutberlet, and G. Scheuermann. Illustrative visualization of cardiac and aortic blood flow from 4D MRI data. In Proc. Pacific Vis, pp. 129-136. IEEE Computer Society, Los Alamitos, 2013. doi> 10.1109/PacificVis.2013.6596137

[4] M. Burns and A. Finkelstein. Adaptive cutaways for comprehensible rendering of polygonal scenes. ACM Transactions on Graphics, 27(5):154:1154:7, 2008. doi $>10.1145 / 1409060.1409107$

[5] J. R. Cebral, M. A. Castro, S. Appanaboyina, C. M. Putman, D. Millan, and A. F. Frangi. Efficient pipeline for image-based patient-specific analysis of cerebral aneurysm hemodynamics: Technique and sensitivity. IEEE Transactions on Medical Imaging, 24(4):457-467, 2005. doi>10.1109/TML. 2005.844159

[6] J. R. Cebral, F. Mut, J. Weir, and C. M. Putman. Association of hemodynamic characteristics and cerebral aneurysm rupture. American Journal of Neuroradiology, 32(2):264-270, 2011. doi> 10.3174/ajnr.A2274

[7] J. Diepstraten, D. Weiskopf, and T. Ertl. Interactive cutaway illustrations. Computer Graphics Forum, 22(3):523-532, 2003. doi> 10.1111/1467-8659. t01-3-00700

[8] M. H. Everts, H. Bekker, J. B. Roerdink, and T. Isenberg. Depth-dependent halos: Illustrative rendering of dense line data. IEEE Transactions on Visualization and Computer Graphics, 15(6):1299-1306, 2009. doi> 10. 1109/TVCG.2009.138

[9] M. H. Everts, H. Bekker, J. B. Roerdink, and T. Isenberg. Illustrative line styles for flow visualization. In Proc. Pacific Graphics, pp. 105-110. IEEE Computer Society, Los Alamitos, 2011. doi> 10.2312/PE/PG/PG2011short/105 $-110$

[10] M. H. Everts, H. Bekker, J. B. T. M. Roerdink, and T. Isenberg. Interactive illustrative line styles and line style transfer functions for flow visualization. Technical Report 1503.05787, arXiv.org, Mar. 2015.

[11] J. Frösen, A. Piippo, A. Paetau, M. Kangasniemi, M. Niemelä, J. Hernesniemi, and J. Jääskeläinen. Remodeling of saccular cerebral artery aneurysm wall is associated with rupture: Histological analysis of 24 unruptured and 42 ruptured cases. Stroke, 35(10):2287-2293, 2004. doi> 10.1161/01.STR.0000140636.30204.da

[12] A. Gambaruto and A. João. Flow structures in cerebral aneurysms. Computers \& Fluids, 65(0):56-65, 2012. Sixth International Conference on Computational Fluid Dynamics (ICCFD6)

[13] R. Gasteiger. Visual Exploration of Cardiovascular Hemodynamics. $\mathrm{PhD}$ thesis, Otto-von-Guericke University Magdeburg, Germany, 2014.

[14] R. Gasteiger, M. Neugebauer, O. Beuing, and B. Preim. The FlowLens: A focus-and-context visualization approach for exploration of blood flow in cerebral aneurysms. IEEE Transactions on Visualization and Computer Graphics, 17(12):2183-2192, 2011. doi> 10.1109/TVCG.2011.243

[15] R. Gasteiger, M. Neugebauer, C. Kubisch, and B. Preim. Adapted surface visualization of cerebral aneurysms with embedded blood flow information In Proc. VCBM, pp. 25-32. Eurographics Association, Goslar, Germany, 2010. doi> 10.2312NCBM/NCBM10/025-032

[16] S. Glaßer, K. Lawonn, T. Hoffmann, M. Skalej, and B. Preim. Combined visualization of wall thickness and wall shear stress for the evaluation of aneurysms. IEEE Transactions on Visualization and Computer Graphics, 20(12):2506-2515, 2014. doi> 10.1109/TVCG.2014.2346406

[17] L. Goubergrits, B. Thamsen, A. Berthe, J. Poethke, U. Kertzscher, K. Affeld, C. Petz, H.-C. Hege, H. Hoch, and A. Spuler. In vitro study of near-wall flow in a cerebral aneurysm model with and without coils. American Journal of Neuroradiology, 31:8:1521 - 1528, 2010.

[18] F. V. Higuera, N. Sauber, B. Tomandl, C. Nimsky, G. Greiner, and P. Hastreiter. Automatic adjustment of bidimensional transfer functions for direct volume visualization of intracranial aneurysms. In Proc. SPIE Medical Imaging, pp. 275-284. SPIE/IS\&T, Bellingham, Washington, 2004. doi> $10.1117 / 12.535534$

[19] L. M. Kadasi, W. C. Dent, and A. M. Malek. Cerebral aneurysm wall thickness analysis using intraoperative microscopy: Effect of size and gender on thin translucent regions. Journal of NeuroInterventional Surgery, 5(3):201-206, 2013. doi > 10.1136/neurintsurg-2012-010285

[20] V. Kajalin. Screen-space ambient occlusion. In W. Engel, ed., Shader $X^{7}$, pp. 413-424. Charles River Media, Boston, 2009.

[21] S. Knödel, M. Hachet, and P. Guitton. Interactive generation and modification of cutaway illustrations for polygonal models. In Proc. Smart Graphics, pp. 140-151. Springer, Heidelberg/Berlin, 2009. doi> 10.1007/ 978-3-642-02115-2_12

[22] K. Lawonn, R. Gasteiger, and B. Preim. Adaptive surface visualization of vessels with animated blood flow. Computer Graphics Forum, 33(8):1627, 2014. doi> 10.1111/cgf.12355
[23] J. Lazar, J. H. Feng, and H. Hochheiser. Interviews and focus groups. In Research Methods in Human-Computer Interaction, chap. 8, pp. 177-216. John Wiley \& Sons Ltd, 2010.

[24] W. Li, L. Ritter, M. Agrawala, B. Curless, and D. Salesin. Interactive cutaway illustrations of complex 3D models. ACM Transactions on Graphics, 26(3):31:1-31:11, 2007. doi> 10.1145/1276377.1276416

[25] R. Liang, G. Clapworthy, M. A. Krokos, and R. Mayoral. Real-time predefined shape cutaway with parametric boundaries. In Proc. Computer Graphics, Imaging and Vision, pp. 227-231, 2005. doi> 10.1109/CGIV.2005.64

[26] E. M. Lidal, H. Hauser, and I. Viola. Design principles for cutaway visualization of geological models. In Proc. SCCG, pp. 47-54. ACM, New York, 2012. doi $>10.1145 / 2448531.2448537$

[27] M. Markl, A. Frydrychowicz, S. Kozerke, M. Hope, and O. Wieben. 4D flow MRI. Journal of Magnetic Resonance Imaging, 36(5):1015-1036, 2012. doi $>10.1002 / \mathrm{mri} .23632$

[28] O. Mattausch, T. Theuß1, H. Hauser, and E. Gröller. Strategies for interactive exploration of 3D flow using evenly-spaced illuminated streamlines. In Proc. SCCG, pp. 230-241. ACM, New York, 2003. doi> 10.1145/984952. 984987

[29] T. Mönch, K. Lawonn, C. Kubisch, R. Westermann, and B. Preim. Interactive mesh smoothing for medical applications. Computer Graphics Forum, 32(8):110-121, 2013. doi> 10.1111/cgf.12165

[30] T. Mönch, M. Neugebauer, and B. Preim. Optimization of vascular surface models for computational fluid dynamics and rapid prototyping. In Proc. International Workshop on Digital Engineering, pp. 16-23, 2011.

[31] M. Neugebauer, R. Gasteiger, O. Beuing, V. Diehl, M. Skalej, and B. Preim. Map displays for the analysis of scalar data on cerebral aneurysm surfaces. Computer Graphics Forum, 28(3):895-902, 2009. doi> 10.1111/.j1467-8659. 2009.01459.x

[32] M. Neugebauer, K. Lawonn, P. Berg, G. Janiga, O. Beuing, and B. Preim. AmniVis - A system for qualitative exploration of near-wall hemodynamics in cerebral aneurysms. Computer Graphics Forum, 32(3):251-260, 2013. doi $>10.1111 / \mathrm{cgf} .12112$

[33] C. Pindat, E. Pietriga, O. Chapuis, and C. Puech. Drilling into complex 3D models with Gimlenses. In Proc. VRST, pp. 223-230. ACM, New York, 2013. doi $>10.1145 / 2503713.2503714$

[34] B. Preim and C. Botha. Visual Computing for Medicine. Morgan Kaufmann, Amsterdam, $2^{\text {nd }}$ ed., 2013. doi $>10.1016 / B 978-0-12-415873-3.00023-7$

[35] J. Raymond, D. Roy, A. Weill, F. Guilbert, T. Nguyen, A. J. Molyneux, A. J. Fox, S. C. Johnston, J.-P. Collet, and I. Rouleau. Unruptured intracranial aneurysms and the trial on endovascular aneurysm management (TEAM): The principles behind the protocol. Journal of Vascular and Interventional Neurology, 1(1):22-26, 2008.

[36] G. Rong and T.-S. Tan. Jump flooding in GPU with applications to Voronoi diagram and distance transform. In Proc. I3D, pp. 109-116. ACM, New York, 2006. doi> 10.1145/1111411.1111431

[37] J. Schöberl. Netgen: An advancing front 2D/3D-mesh generator based on abstract rules. Computing and Visualization in Science, 1(1):41-52, 1997. doi> 10.1007/5007910050004

[38] S. Sigg, R. Fuchs, R. Carnecky, and R. Peikert. Intelligent cutaway illustrations. In Proc. PacificVis, pp. 185-192. IEEE Computer Society, Los Alamitos, 2012. doi> 10.1109/PacificVis.2012.6183590

[39] C. Teitzel, R. Grosso, and T. Ertl. Efficient and reliable integration methods for particle tracing in unsteady flows on discrete meshes. In Proc. Visualization in Scientific Computing, pp. 31-41. Springer, Berlin/Heidelberg, 1997. doi>10.1007/978-3-7091-6876-9 4

[40] R. van Pelt, J. O. Bescos, M. Breeuwer, R. E. Clough, M. E. Groller, B. ter Haar Romenij, and A. Vilanova. Exploration of 4D MRI blood flow using stylistic visualization. IEEE Transactions on Visualization and Computer Graphics, 16(6):1339-1347, 2010. doi> 10.1109/TVCG.2010.153

[41] R. van Pelt, J. O. Bescós, M. Breeuwer, R. E. Clough, M. E. Gröller, B. M. ter Haar Romeny, and A. Vilanova. Interactive virtual probing of 4D MRI blood-flow. IEEE Transactions on Visualization and Computer Graphics, 17(12):2153-2162, 2011. doi> 10.1109/TVCG.2011.215

[42] A. Vilanova, B. Preim, R. van Pelt, R. Gasteiger, M. Neugebauer, and T. Wischgoll. Visual exploration of simulated and measured blood flow. In Scientific Visualization, pp. 305-324. Springer, Berlin/Heidelberg, 2014. doi $>10.1007 / 978-1-4471-6497-525$

[43] I. Viola, A. Kanitsar, and M. E. Gröller. Importance-driven volume rendering. In Proc. IEEE Visualization, pp. 139-145. IEEE Computer Society, Los Alamitos, 2004. doi> 10.1109NISUAL.2004.48 